\title{
EFECTO DEL ÁNGULO DE INCIDENCIA SOBRE MONOCAPAS DE HfN EXPUESTAS A FENÓMENOS DE CORROSIÓN EROSIÓN
}

\section{EFFECT OF THE ANGLE OF INCIDENCE ON HFN MONOLAYERS EXPOSED TO CORROSION EROSION PHENOMENA}

Pablo Andrés Guzmán Durán', William Aperador Chaparro², José Luis Caballero Gómez³

Fecha de recepción: 29 de Septiembre de 2014

Fecha de aprobación: 24 de Diciembre de 2014

Referencia: P.A. Guzmán Durán, W.A. Chaparro, J.L. Caballero Gómez. (2015). Efecto del ángulo de incidencia sobre monocapas de HfN expuestas a fenómenos de corrosión-erosión. Ciencia e Ingeniería Neogranadina, 25 (1), pp. 5 - 15

\section{RESUMEN}

Los recubrimientos duros son una alternativa para el mejoramiento superficial de herramientas industriales, ya que son desarrollados con el fin de aumentar la vida de servicio del material mediante un mejoramiento de sus características frente a mecanismos de desgaste y fenómenos corrosivos. En el presente estudio se depositaron monocapas de nitruro de hafnio sobre sustratos de acero AISI 4140 mediante la técnica del magnetrón sputtering multi-blanco en r.f. (13.56 MHz). Esto se hizo con el objetivo de determinar valores estimados de la pérdida de material, el desgaste mecánico y la sinergia en los fenómenos corrosivos y erosivos con base en la norma ASTM G119-03, que interrelaciona la corrosión con el desgaste. Las monocapas fueron evaluadas frente a fenómenos de corrosión-erosión, erosión y corrosión a dos ángulos de impacto de $30^{\circ}$ y $90^{\circ}$, en una solución compuesta por $\mathrm{NaCl} 0.5 \mathrm{M}$ usando un equipo de incidencia de chorro de partícula. Se analizó el efecto del ángulo de impacto en la resistencia a la corrosión erosión de estos recubrimientos. Mediante curvas de polarización Tafel y microscopia electrónica de barrido se realizó la evaluación electroquímica y la caracterización micro-estructural de los recubrimientos respectivamente. Se observó un aumento en la velocidad de corrosión para los sistemas sometidos a $90^{\circ}$ y una disminución para los sistemas a $30^{\circ}$.

1. Ing. Mecatrónico, Asistente de investigación, Facultad de Ingeniería. Universidad Militar Nueva Granada, Bogotá, Colombia, pabloaguzman88@ gmail.com

2. Físico, Ph.D. en Ingeniería de materiales, Facultad de Ingeniería. Universidad Militar Nueva Granada, Bogotá, Colombia, william.aperador@unimilitar.edu.co

3. Ing. Mecatrónico, Especialista en Gerencia Integral de Proyectos, Asistente Graduado, Facultad de Ingeniería. Universidad Militar Nueva Granda, Bogotá, Colombia, caballerojl22@gmail.com 
Palabras clave: Sinergia, corrosión, erosión.

\begin{abstract}
Hard coatings are an alternative for surface improvement of industrial tools since they are developed with the purpose of increasing the lifespan of materials through the enhancement of its characteristics against wear mechanisms and corrosion phenomena. In this study, hafnium nitride monolayers were deposited on AISI 4140 steel substrates through the RF magnetron sputtering multi-target technique (13.56 MHz) to determine estimated values of material loss, mechanical wear and synergy in the corrosion and erosion phenomena, according to the ASTM G119-03 standard, which relates corrosion to wear. The monolayers were evaluated against corrosion-erosion phenomena, as well as corrosion and erosion at two incidence angles of $30^{\circ}$ and $90^{\circ}$ in a solution composed by $0.5 \mathrm{M} \mathrm{NaCl}$, using impingement jet equipment to analyze the effect of the incidence angle on the corrosion erosion resistance of these coatings. Through Tafel polarization curves and scanning electron microscopy, the electrochemical evaluation and the micro-structural characterization of the coatings were respectively made. An increase in the corrosion rate on the systems subjected to $90^{\circ}$ and a decrease on those subjected to $30^{\circ}$ was observed.
\end{abstract}

Keywords: Synergy, corrosion, erosion.

\section{INTRODUCCIÓN}

Fenómenos como el desgaste y la corrosión son problemas a los que se ven enfrentados frecuentemente dispositivos de máquinas industriales, en especial los relacionados con el transporte de fluidos como partes de motobombas y aglutinadoras de polímeros [1]. Esto se debe a que algunos de los fluidos son sustancias agresivas que, además, contienen partículas en suspensión, lo que genera dos fenómenos combinados, la corrosión y la erosión, ocasionando grandes daños a las partes expuestas y acortando drásticamente la vida útil de los elementos [2].

Las películas delgadas en forma de monocapas [3], son materiales objeto de estudio debido a que, con la deposición de éstas, pueden mejorarse propiedades tales como la resistencia a la corrosión y al desgaste, la conductividad eléctrica, y utilizarse como barreras de difusión [3]. Con la deposición de varias capas con diferentes propiedades mecánicas entre ellas, se pueden controlar problemas como tensiones superficiales y propagación de fisuras [4].

Los recubrimientos duros han sido diseñados para aplicaciones en las que el acero tradicionalmente presenta daños [5], como es el caso del desgaste que se produce por el impacto continuo de partículas y la degradación por el contacto con fluido corrosivo. Los recubrimientos por deposición física en fase de vapor (PVD) [3], ofrecen ventajas de rendimiento en aplicaciones, ya que ofrecen una adecuada protección contra 
el desgaste. Estos recubrimientos pueden ser clasificados de acuerdo al carácter del enlace químico en los siguientes grupos: materiales duros iónicos, covalentes y metálicos. Esta clasificación contribuye considerablemente a la comprensión de las diferentes estructuras cristalinas y del comportamiento de los materiales en forma de capas delgadas simples.

El nitruro de hafnio (HfN) tiene como principal aplicación el área de recubrimientos que funcionan como barreras térmicas a altas temperaturas [6]. Adicionalmente, posee una dureza superior a los carburos y otros nitruros como lo son el TiN y CrN [7-8]. Por esta razón, las películas delgadas de HfN [9], son un excelente material protector para herramientas de corte de alta velocidad [10]. Según la estequiometria del HfN, este es uno de los compuestos más estables entre los mononitruros de los metales de transición a altas temperaturas, lo cual lo convierte en un candidato ideal como barrera de difusión en esquemas de metalización. La ventaja del HfN es que presenta poca influencia sobre propiedades como dureza y esfuerzos internos, frente a variables de deposición como el voltaje bias.

La corrosión-erosión es una aceleración en la velocidad de corrosión en un metal debido al movimiento relativo de un fluido corrosivo y la superficie del metal. Si además el fluido presenta contenido de partículas sólidas en suspensión, la tendencia es a incrementar el efecto erosivo causando deterioro de la superficie metálica.

Los recubrimientos duros se han convertido en la solución a problemas como la corrosión y el desgaste [11]. La técnica PVD es uno de los procesos más utilizados para la obtención de recubrimientos duros, englobando cualquier proceso de crecimiento de recubrimientos en un ambiente de vacío que implique la deposición de átomos o moléculas en un sustrato. Esta técnica permite evaporar por medios físicos el material que formará el recubrimiento y condensarlo sobre el sustrato. Este proceso tiene la ventaja de poder aplicarse simultáneamente a conjuntos o piezas.

En este trabajo se estudió la sinergia entre la corrosión dinámica, erosión y corrosión-erosión de los recubrimientos tipo monocapas basados en HfN expuestos a un medio agresivo en condiciones dinámicas. El presente estudio tiene como objetivo evaluar los recubrimientos de $\mathrm{HfN}$ con respecto a la resistencia al desgaste frente a la erosióncorrosión, al determinar la mejoría en cuanto a estos fenómenos en comparación con los aceros AISI 4140 [12], e indicar un mejor desempeño con respecto a la sinergia del desgaste mecánico y la degradación electroquímica al variar el efecto del impacto de las partículas de sílice en dos ángulos $\left(30^{\circ}\right.$ y $\left.90^{\circ}\right)$.

\section{METODOLOGÍA}

\subsection{DEPOSICIÓN FÍSICA EN FASE DE VAPOR}

Monocapas de nitruro de hafnio se depositaron sobre sustratos de acero AISI 4140, los cuales fueron desengrasados por ultrasonido en una secuencia de 15 minutos de etanol y acetona. Los recubrimientos se obtuvieron mediante la técnica del magnetrón sputtering multiblanco en r.f. (13.56 MHz). Para la deposición de los recubrimientos se utilizaron blancos de 4 pulgadas de diámetro de hafnio con una pureza del $99.9 \%$. 
La presión base al interior de la cámara de vacío fue de $2.3 \times 10^{-3}$ mbar. Antes de iniciar la deposición, los sustratos fueron expuestos a una limpieza por plasma durante 15 minutos en atmósfera de Ar a un bias de $-400 \mathrm{~V}$ en r.f. Durante el crecimiento, los gases de trabajo fueron una mezcla de $\operatorname{Ar}(93 \%)$ y $N_{2}(7 \%)$ con una presión total de trabajo de $3.6 \times 10^{-3}$ mbar, a una temperatura del sustrato de $400{ }^{\circ} \mathrm{C}$, un bias r.f. del sustrato de $-70 \mathrm{~V}$ y una potencia de $400 \mathrm{~W}$.

Con el fin de estudiar la influencia de la sinergia entre la corrosión dinámica, erosión y corrosiónerosión de recubrimientos monocapas se depositaron monocapas de HfN. El espesor de los recubrimientos fue obtenido mediante un perfilómetro DEKTAK 8000 con un diámetro de punta de $12 \pm 0.04 \mu \mathrm{m}$ a una longitud de barrido entre $1000-1200 \mu \mathrm{m}$. Para todas las muestras el espesor fue de $3 \mu \pm 0.04 \mu \mathrm{m}$.

\subsection{EQUIPO DE EROSIÓN}

En cuanto a la evaluación de la resistencia a la corrosión-erosión se utilizó un equipo de incidenciadechorrodepartícula, comosemuestra en la Figura 1. Este consiste en un flujo acuoso que se mantiene en movimiento constante por un sistema de recirculación conformado por mangueras, acoples y una bomba de conducción magnética, que controla la velocidad del flujo. La temperatura del fluido es regulada por medio de una resistencia térmica. La cámara de acrílico permite tener las condiciones adecuadas para la realización de los ensayos por ataque directo o por inmersión y el ángulo de incidencia o ángulo de impacto es seleccionado mediante la posición del portaprobeta.

El equipo permite controlar tres variables independientemente: la temperatura del fluido, la velocidad del flujo y el ángulo de impacto. Los ensayos se realizaron con un ángulo de impacto de $30^{\circ}$ y $90^{\circ}$. La velocidad de impacto genera una velocidad lineal promedio de la partícula de $18.5 \mathrm{~m} \mathrm{~s}^{-1}$ y una temperatura de ensayo de $25^{\circ} \mathrm{C}$. El equipo tiene adaptado el electrodo de referencia - ER ( $\mathrm{Ag} / \mathrm{AgCl})$, el contraelectrodo - EA (Alambre de platino) y el portamuestras - ET con un área de exposición de la muestra de $1 \mathrm{~cm}^{2}$. La mezcla se mantiene homogénea debido al movimiento realizado por la bomba mecánica.

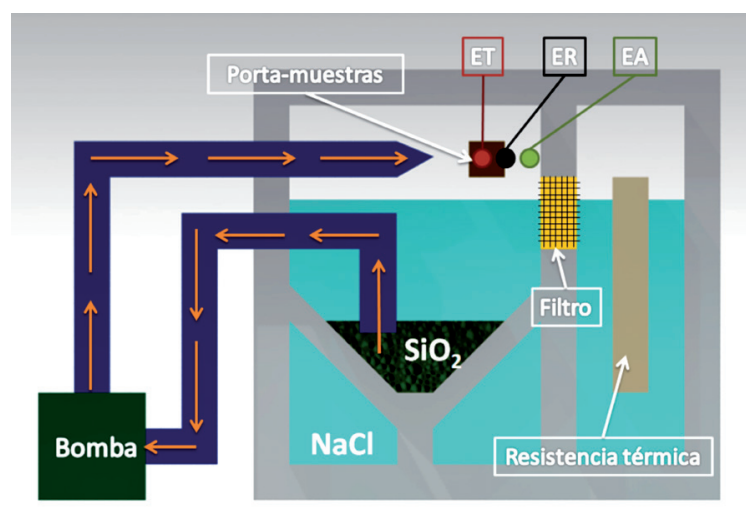

Figura 1. Esquema del equipo utilizado en la prueba de desgaste de la erosión-corrosión, EA: electrodo auxiliar; ET: electrodo de trabajo; ER: electrodo de referencia.

\subsection{EVALUACIÓN DEL RECUBRIMIENTO}

Para la evaluación de la resistencia a la corrosión dinámica y corrosión-erosión se utilizó un potenciostato-galvanostato Gamry modelo PCl-4, mediante la técnica de polarización anódica. Se ubicaron las probetas bajo inmersión en una solución de $\mathrm{NaCl} 0.5$ M preparada con agua destilada y partículas de sílice $\left(\mathrm{SiO}_{2}\right)$ con tamaño de partícula entre $210 \mu \mathrm{m}$ y $300 \mu \mathrm{m}$ bajo una proporción del 
$20 \%$ wt con respecto al medio. La celda fue compuesta por un contraelectrodo (alambre de platino), un electrodo de referencia de $\mathrm{Ag} /$ $\mathrm{AgCl}$ y como electrodo de trabajo se utilizó el acero AISI 4140 con y sin recubrimiento en monocapas. La solución de trabajo permite simular condiciones marinas y además corroe metales activos formando cloruros sobre el metal. Los diagramas de Tafel se obtuvieron a una velocidad de barrido de $0.5 \mathrm{mV} / \mathrm{s}$ en un rango de voltajes de $-0.25 \mathrm{~V}$ a $0.3 \mathrm{~V}$. El comportamiento electroquímico fue evaluado después de 45 minutos, tiempo necesario para la estabilización del potencial de circuito abierto.

Las normas utilizadas en los criterios de medición y cálculos corresponden a las ASTM G3, G5 y G59. Adicionalmente, las muestras fueron expuestas a desgaste por erosión durante un tiempo total de exposición de 1440 minutos a temperatura de $25^{\circ} \mathrm{C}$ en una solución de $\mathrm{NaCl} 0.5 \mathrm{M}$ con partículas de sílice (SiO2). Para determinar la pérdida de peso debido a la erosión, las muestras se retiraron de la solución a intervalos de tiempo de 15 minutos, se limpiaron con un chorro de agua, se secaron con aire caliente y se pesaron en una balanza de precisión (0.1 mg). La evaluación del fenómeno erosivo se realizó utilizando protección catódica de $-1 \mathrm{~V}$ con respecto al potencial de circuito abierto del acero y del recubrimiento, respectivamente, de acuerdo con la norma ASTM G119-93. Esta protección catódica garantizó daño superficial sólo por efecto de las partículas erosivas. Los fenómenos de degradación fueron observados con un microscopio electrónico de barrido (SEM). Tanto la morfología de crecimiento como las características superficiales se determinaron con un SEM Phenom FEI equipado con una luz óptica con un rango de magnificación de 50 - 40 000X.

\section{RESULTADOS Y DISCUSIÓN}

En la Figura 2 se observan las curvas de polarización del acero y los recubrimientos evaluados bajo el medio salino. Con las modificaciones en el ángulo de impacto es posible identificar la prevalencia del mecanismo de desgaste electroquímico en cada caso. Los recubrimientos de monocapas de HfN muestran una diferencia en la densidad de corriente de corrosión al ser evaluados con respecto a diferente energía de impacto debido a las partículas erosivas. En las curvas de polarización se obtiene que los potenciales de corrosión para los dos impactos evaluados en las monocapas son similares. La variación se encuentra en el impacto del fluido y de partículas erosivas.

En los dos ángulos de ataque evaluados se observa que hay mayor daño para el recubrimiento con un impacto de $90^{\circ}$ en comparación al de $30^{\circ}$, debido a la mayor densidad de corriente y menor potencial de corrosión. Se obtiene un aumento de seis veces en la densidad de corriente de corrosión. Esto se debe al efecto del ataque de las partículas de forma rasante, lo cual puede generar desprendimiento de la película delgada. Al observar el comportamiento anódico, se obtiene en los dos mecanismos evaluados una corrosión de forma general, debido a que al incrementar el potencial de corrosión, la densidad de corrosión se incrementa. Al comparar el desempeño de los recubrimientos con el sustrato AISI 4140, se obtiene que el potencial de corrosión mantiene una tendencia a ser noble para el recubrimiento en los dos mecanismos evaluados, con una diferencia de potencial en promedio de +200 $\mathrm{mV}$ con respecto al sustrato, lo que genera una protección frente a fenómenos corrosivos y erosivos. Al comparar la densidad de corriente 
de corrosión, se obtiene un incremento para el sustrato. Esto se debe a que la capa de protección pasiva se diluye gracias al efecto de la sinergia, por lo que el efecto de degradación es más acelerado para el sustrato en contraste al mecanismo observado para las Monocapas. Lo anterior se debe a que la densidad disminuye 25 veces menos que el acero AISI 4140, lo que indica que el efecto protector es adecuado utilizando los recubrimientos de HfN.

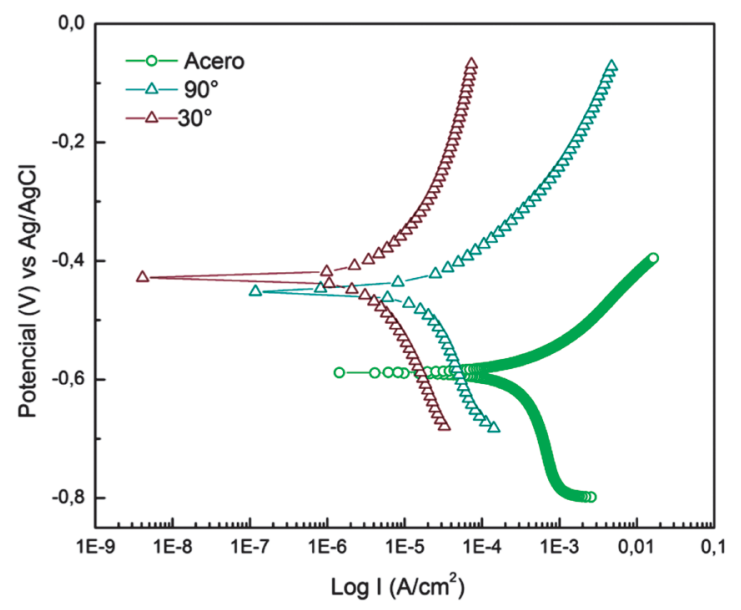

Figura 2. Curvas de polarización de los recubrimientos monocapas de HfN evaluadas en los ángulos de $30^{\circ}$ y $90^{\circ}$. Se incluye el acero a $90^{\circ}$ para comparar el sistema.

En la Tabla 1 se reportan en forma cualitativa los valores de los parámetros electroquímicos que permiten indicar el desempeño de las monocapas para los dos ángulos de impacto $30^{\circ}$ y $90^{\circ}$. Los recubrimientos muestran una disolución del material de forma moderada. Este fenómeno se encuentra en todos los recubrimientos a $-420 \mathrm{mV}$ vs. $\mathrm{Ag} / \mathrm{AgCl}$, lo que indica la regeneración de capas de productos de corrosión que permiten estabilizar la densidad de corriente en torno a este potencial y prevenir el incremento de la velocidad de disolución del metal.
Tabla 1. Parámetros electroquímicos que muestran el desempeño de las monocapas

\begin{tabular}{|c|c|c|c|}
\hline Parámetros & Acero & $9^{\circ}$ & $\mathbf{3 0}^{\circ}$ \\
\hline $\begin{array}{c}\text { Potencial de } \\
\text { corrosión (mV) }\end{array}$ & $-590,23$ & $-431,38$ & $-454,94$ \\
\hline $\begin{array}{c}\text { Corriente de } \\
\text { corrosión } \\
\left(\mu \mathrm{A} / \mathrm{cm}^{2}\right)\end{array}$ & 388 & 18,10 & 3,62 \\
\hline $\begin{array}{c}\text { Velocidad de } \\
\text { corrosión (mpy) }\end{array}$ & 171,3 & 7,99 & 1,59 \\
\hline
\end{tabular}

La Figura 3 muestra los diagramas de Nyquist del sustrato y los recubrimientos monocapas sumergidos en solución de $\mathrm{NaCl}$ al $0.5 \mathrm{M}$ y expuestas a corrosión dinámica con adición de partículas erosivas. Estos recubrimientos presentan un comportamiento de aumento de la impedancia total en cada una de las monocapas evaluadas, siendo más sobresaliente el evaluado a los $30^{\circ}$. Los diagramas Nyquist correspondientes a las monocapas muestran un comportamiento capacitivo a elevadas frecuencias, en el que se define un semicírculo aplanado. Este fenómeno de aplanamiento del semicírculo se asocia con un proceso de dispersión en la frecuencia, debido a que la superficie del electrodo no es homogénea. Adicionalmente, se observa un proceso de difusión que pretende definir un segundo semicírculo a bajas frecuencias (Figura 3b).

En cada uno de los espectros analizados se incluyen los resultados de la simulación mediante el circuito eléctrico que se muestra en la Figura 4. Como se puede observar, hay una buena concordancia entre los resultados experimentales y los simulados. En la Figura 4 se observa el circuito equivalente correspondiente a los diagramas de Nyquist 


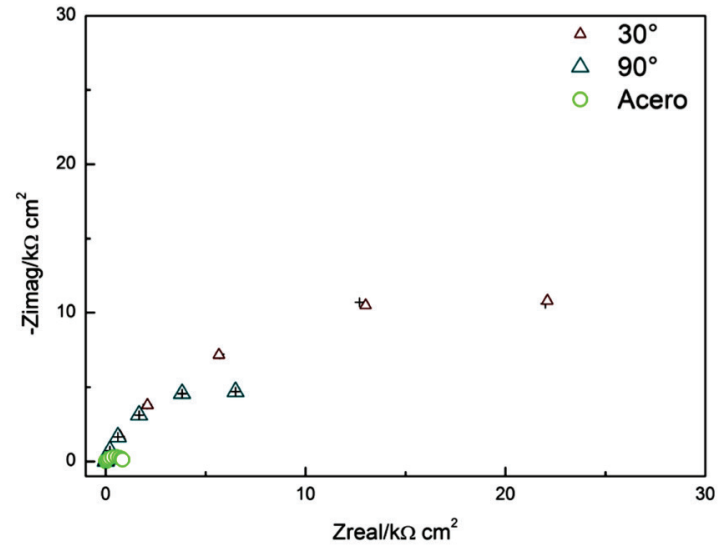

(a)

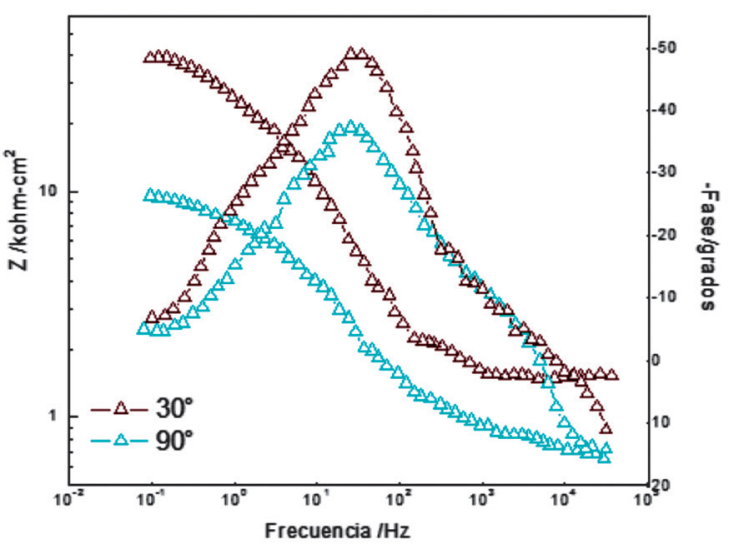

(b)

Figura 3. a) Diagramas de impedancia Nyquist y b) Diagramas de bode del acero y las monocapas de HfN sometidas a corrosión dinámica con partículas de silice para los ángulos de $30^{\circ}$ y $90^{\circ}$.

para los recubrimientos, los cuales muestran una capacitancia denominada «elemento de fase constante» (CPE), que es independiente de reacciones farádicas, las cuales contribuyen con una pseudocapacitancia (CPE2+CPE1) a la impedancia total del sistema. Por otra parte, en esta celda electroquímica existe también una resistencia eléctrica asociada a la resistencia del electrolito (Rs).

Para la muestra de acero AISI 4140 (sustrato) se obtiene un pequeño semicírculo insignificante comparado con los resultados de los recubrimientos monocapas de HfN. El modelo que proporcionó mejor ajuste es el presentado en la Figura 4a. En este circuito se observa una constante de tiempo conectada en paralelo con la resistencia a la transferencia de carga, y en serie con la resistencia de la solución. Para los recubrimientos en los cuales se varían los ángulos de impacto se observan dos semicírculos, por esta razón se utilizan dos elementos de fase constante (CP1 y CP2) (ver Figura 4b) para modelar un

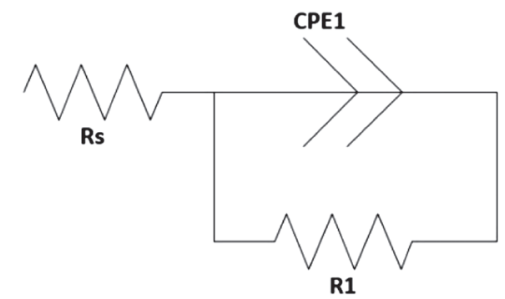

a)

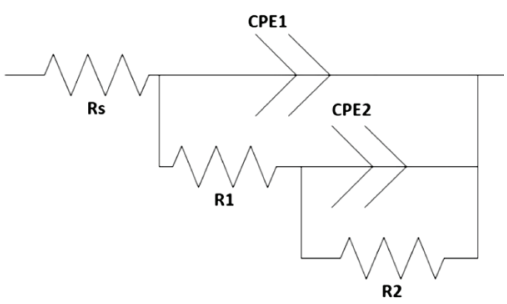

b)

Figura 4. Circuito equivalente. a) Circuito para ajustar los datos de impedancia del sustrato; b) Circuito para ajustar los datos de impedancia de los recubrimientos con los dos números de ángulos de impacto. 
circuito equivalente. Los elementos CP1-R1, se presentan a altas frecuencias y se asocian a las reacciones que ocurren alrededor de la capa de óxido superficial pasivante generado por los recubrimientos. Un segundo conjunto de elementos CP2-R2, presentes a muy bajas frecuencias $(1 \mathrm{mHz}$ ) están relacionados con la capa barrera generada por la capa de óxido superficial y el sustrato debido a la transferencia de carga. Este conjunto de elementos representa la respuesta de los procesos ocurridos en el sistema, los cuales son lentos en los recubrimientos obtenidos.

\subsection{EFICIENCIA DE LOS RECUBRIMIENTOS}

Con los ensayos de caracterización electroquímica se puede determinar la eficiencia de protección, EP (\%), de los recubrimientos por medio de la ecuación (1):

$$
E P(\%)=\left[1-\left(\frac{i_{\text {corr }}}{i_{\text {corr }}^{0}}\right)\right] \cdot 100
$$

donde $\mathrm{i}_{\text {corr }}$ e $\mathrm{i}^{0}$ corr indican la densidad de corriente del recubrimiento y del sustrato, respectivamente. Las eficiencias de protección calculadas y las resistencias de polarización se presentan en la Figura 5. La capacidad protectora de la película aumenta con la disminución del ángulo de impacto. Así, el recubrimiento evaluado a $30^{\circ}$ presenta el mayor efecto de protección (99.06\%), causada por la menor densidad de corriente de $3.62 \mu \mathrm{A} / \mathrm{cm}^{2}$.

El valor de la porosidad del recubrimiento se midió cuantitativamente, dado que la porosidad corresponde a la relación entre la resistencia a la polarización del substrato y del recubrimiento, como se muestra en la ecuación (2):

$$
P=\frac{R_{p-s}}{R_{p-r}}
$$

donde Pes la porosidad total del recubrimiento, $\mathrm{R}_{(\mathrm{p}-\mathrm{s})}$ es la resistencia a la polarización del substrato y $\mathrm{R}_{(\mathrm{p}-\mathrm{r})}$ la resistencia a la polarización del recubrimiento. El valor correspondiente del recubrimiento evaluado $90^{\circ}$ es de $6.57 \%$ y para $30^{\circ}$ el valor corresponde a $18.04 \%$. Lo anterior se debe a que la deposición de moléculas de nitruro de hafnio genera una estructura densa libre de poros. Sin embargo, las características electroquímicas en combinación con el efecto mecánico generan aumento en la porosidad, lo que produce pérdida de la eficiencia del recubrimiento, en especial a ángulos rasantes.

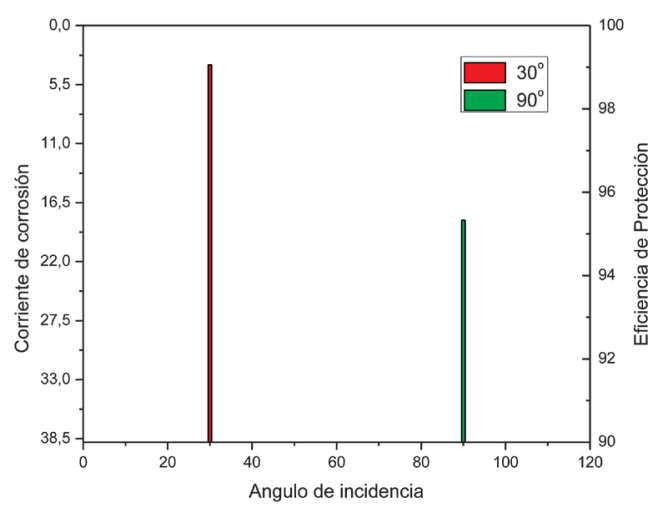

Figura 5. Corriente de corrosión y eficiencia de protección.

\subsection{EROSIÓN}

Para realizar un análisis del efecto de la erosión, en la Figura 6 se muestra la perdida de 
material causada por los ángulos de incidencia de $30^{\circ}$ y $90^{\circ}$. En la gráfica se observa cómo un mayor ángulo de impacto $\left(90^{\circ}\right)$ produce una mayor pérdida de masa. Inicialmente se tiene una alta pérdida de masa para los dos ángulos de incidencia, pero con el transcurso del tiempo se puede notar cómo, al tener tiempos de estabilización similares en la curva de pérdida de masa, finalmente los niveles son más bajos para ángulos menores.

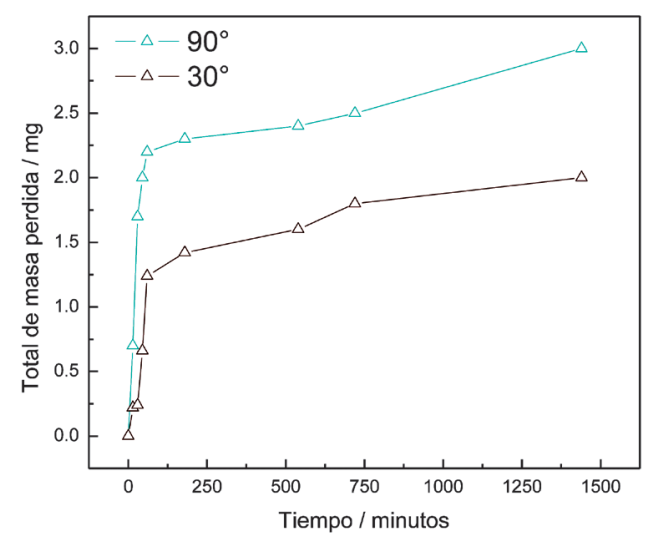

Figura 6. Curvas de pérdida de masa por el efecto erosión.

\subsection{MICROSCOPIA ELECTRÓNICA DE BARRIDO}

En las micrografías de la Figura 7 se observan las características superficiales de las monocapas de nitruro de hafnio luego del proceso de corrosión-erosión a un ángulo de impacto de $30^{\circ}$ y $90^{\circ}$. Según la Figura $7 \mathrm{a}$, correspondiente a la probeta evaluada a $90^{\circ}$, el recubrimiento presenta un deterioro como consecuencia del efecto de la corrosión dinámica y las partículas que han generado forma de surco. Esto es debido a que las partículas generan una abrasión sobre la película delgada.

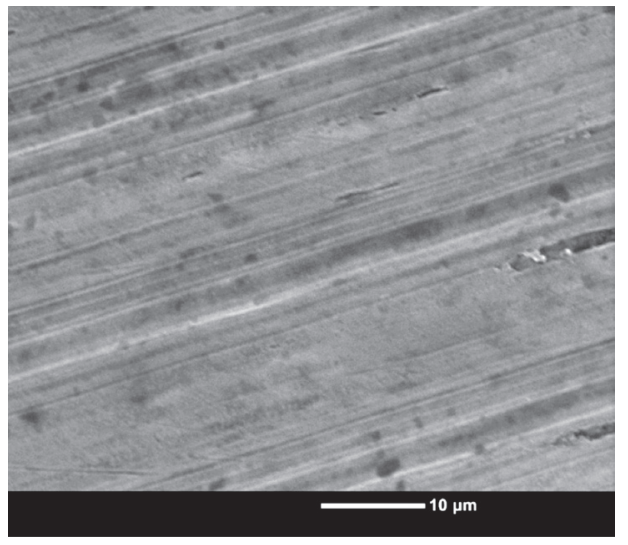

a)

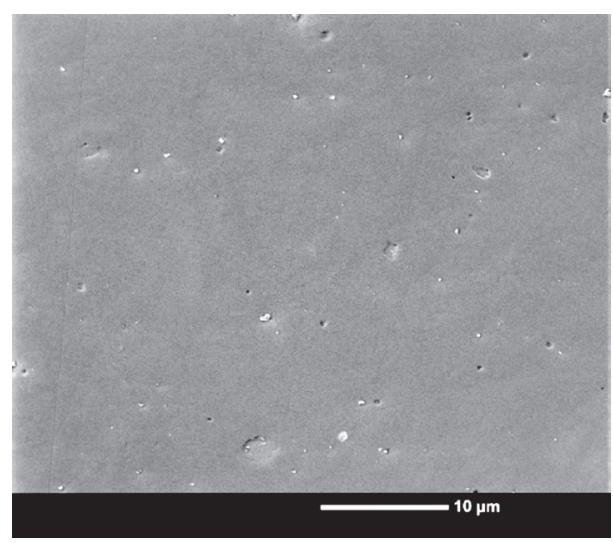

b)

Figura 7. a) Micrografía de la probeta evaluada con un ángulo de impacto de $90^{\circ}$ b) Micrografía de la probeta evaluada con un ángulo de impacto de $30^{\circ}$.

En la figura 7b se observa una zona donde el daño por procesos corrosivos no es visible. Al exponer estos recubrimientos a $30^{\circ}$, se genera un sistema de protección y se obtienen regiones en donde el impacto de las partículas genera daño mecánico sin producirse agrietamiento. Estas áreas muestran una superficie libre de fracturas representando la acción protectora otorgada por los recubrimientos bajo esta condición, que se debe a la eliminación de la capa protectora por la acción erosiva. 


\section{CONCLUSIONES}

Teniendo en cuenta el ángulo de incidencia, fue posible determinar cómo brindar una mejor protección frente a la corrosión. La eficiencia de protección del ángulo de $90^{\circ}$ fue menor en comparación a la del ángulo de $30^{\circ}$, lo que permite identificar la correlación que existe entre los ángulos de incidencia y la eficiencia de protección frente a fenómenos erosivos.

En las micrografías de los dos diferentes ángulos de impacto se puede observar el efecto que tiene el recubrimiento como capa protectora ante la erosión. Se obtuvo capa más gruesa para $90^{\circ}$, en la que solo se observa impactos de las partículas en la superficie pero con mayores daños corrosivos, mientras que para $30^{\circ}$ se puede visualizar un micro-arado como consecuencia del desgaste pero con procesos corrosivos menores.

\section{AGRADECIMIENTOS}

Producto derivado del Proyecto ING1526 financiado por la Vicerrectoría de Investigaciones de la UMNG vigencia 2014.

\section{REFERENCIAS BIBLIOGRÁFICAS}

[1] Efremenko, V. G., Shimizu, K., Noguchi, T., Efremenko, A. V. y Chabak, Y. G. (2013). Impact-abrasive-corrosion wear of $\mathrm{Fe}$ based alloys: Influence of microstructure and chemical composition upon wear resistance. Wear, 305(1-2), 155-165.

[2] Papavinasam, S. (2014). Corrosion Control in the Oil and Gas Industry. San Diego, EE.UU: Gulf Professional Publishing.
[3] Sarkar, J. (2014). Sputtering Materials for VLSI and Thin Film Devices (pp.93-170). Oxford, Reino Unido: Elsevier.

[4] Zhang, W., Pommier, S., Curtit, F., Léopold, G. y Courtin, S. (2014). Mode I Crack Propagation under High Cyclic Loading in 316L Stainless Steel. Procedia Mater. Sci., 3, 1197-1203, 2014.

[5] Pereira, D., Gandra, J., Pamies-Teixeira, J., Miranda, R.M. y Vilaça, P. (2014). Wear behaviour of steel coatings produced by friction surfacing. J. Mater. Process. Technol., 214(12), 2858-2868.

[6] Escobar, C., Villarreal, M., Caicedo, J. C., Aperador, W. y Prieto, P. (2013). Novel performance in physical and corrosion resistance HfN/VN coating system. Surf. Coatings Technol., 221, 182-190.

[7] Nordin, M. y Larsson, M. (1999). Deposition and characterization of multilayered PVD TiN/CrN coatings on cemented carbide. Surf. Coatings Technol., 116-119, 108-115.

[8] Ruden, A., Restrepo-Parra, E., Paladines, A.U. y Sequeda, F. (2013). Corrosion resistance of $\mathrm{CrN}$ thin films produced by dc magnetron sputtering, Appl. Surf. Sci., 270, 150-156.

[9] Staia, M.H., Bhat, D.G., Puchi-Cabrera, E.S. y Bost, J. Characterization of chemical vapor deposited HfN multilayer coatings on cemented carbide cutting tools. Wear, 261(5-6), 540-548.

[10] Liu, Z., Ai, X., Zhang, H., Wang, Z. y Wan, Y. (2002). Wear patterns and mechanisms of cutting tools in high-speed face milling. J. Mater. Process. Technol., 129(1-3), 222-226. 
[11] Jehn, H. A. (2000). Improvement of the corrosion resistance of PVD hard coating-substrate systems. Surf. Coatings Technol., 125(1-3), 212217.
[12] Ulutan, M., Celik, O.N., Gasan, H. y Er, U. (2010). Effect of Different Surface Treatment Methods on the Friction and Wear Behavior of AISI 4140 Steel. J. Mater. Sci. Technol., 26(3), 251-257. 